\title{
Queer sentences, ambiguity, and levels of processing
}

\author{
JANET L. MISTLER-LACHMAN \\ The University of Houston, Houston, Texas 77004
}

\begin{abstract}
Subjects judged linguistic strings "meaningful" or "meaningless." Meaningful sentences were identical for all subjects; however, for each of five groups, meaningless foils containing different kinds of linguistic violation were interspersed among the meaningful sentences. Type of foil influenced processing time for meaningful items, suggesting that laboratory language processing may be determined by the entire set of linguistic materials used. Effect of foil type on comprehension depth for meaningful items was assessed from the extent to which three kinds of ambiguity slowed judgments on those items as compared to unambiguous sentences. Foil type appears to affect depth of meaningful sentence processing in such a way as to support a "levels of analysis" view of sentence comprehension. Foil type and kind of ambiguity interacted to suggest that sentence comprehension requires computation of underlying logical relationships prior to computation of surface structural relationships and the unequivocal determination of word meanings.
\end{abstract}

Craik and Lockhart (1972) have proposed an alternative to the multistore model, which focuses on encoding levels, rather than stores. While the Craik and Lockhart model is not necessarily inconsistent with multistore formulations, the focus is clearly different. Craik and Lockhart view encoding level as a function of task demands, properties of stimulus material, and the like. Where multistore models might view additional depth as stages or subprocesses added on to the end of a processing sequence, in Craik and Lockhart's view, different task demands precipitate different levels of encoding from the beginning of processing, raising the possibility that deeper processing need not necessarily take longer. Craik and Lockhart argue that more "semantic" tasks lead to deeper encoding levels. A direct extension of their argument is that a task requiring greater elaboration of sentence meaning will lead to deeper encoding levels for the material.

The foregoing considerations raise interesting substantive and methodological questions for both multistore and levels-of-encoding models. First, what qualitative differences can be identified in depth of comprehension when all tasks involve semantic processing? Second, can depth of comprehension be changed by varying the nature of the stimulus materials used in a single task? If the nature of the stimulus materials influences depth of processing, has our choice of laboratory materials kept us from observing typical extralaboratory performance in similar tasks? Put another way, is the depth of processing adopted by a laboratory subject significantly determined by the particular set of materials chosen by the experimenter?

The present experiment was designed to vary the depth of comprehension required for a subject to determine whether a linguistic string is meaningful by

This research was supported by Kansas University General Research Fund Grant No. 3303. The author thanks Earl Butterfield, Roy Lachman, and James Juola for their helpful comments and suggestions. varying the nature of meaningless foils. The logic is similar to that of Mistler-Lachman (1972). In that study, subjects performed three information-processing tasks. In two of the tasks, processing was slowed when stimulus sentences were ambiguous. In the third task, ambiguity did not affect processing time. Mistler-Lachman inferred that the task required no ambiguity resolution, and was therefore shallower than the other tasks. The present experiment uses this shallow task, which requires subjects to judge a sentence as meaningful or anomalous. Mistler-Lachman used anomalous strings which were quite bizarre: e.g., the spool of the calamity was not pointed until footprints. The meaningfulness judgment task might have required deeper comprehension if the violations of English syntactic rules in the foils had been less extensive. The present study utilizes five different types of rule violation. Two conditions involving the grossest violations involve first, scrambling the words of a normal sentence and second, violating several English linguistic rules in each string, while retaining English word order. The other three conditions involve progressively less severe violations of English grammatical rules, according to an order suggested by Chomsky (1965): Phrase marker violations, subcategorization rule violations, and selectional restriction violations. Phrase marker violations are constructed by changing a word in a normal sentence to another word of the wrong part of speech. The remaining two conditions are accomplished by violating the subcategorization rules and selectional restrictions of English, respectively. Table 1 contains examples of each violation condition. Several studies [Hamilton \& Deese (Note 1); Moore, 1972; Stolz, 1969] have found Chomsky's ordering of linguistic violations in terms of severity to predict performance on laboratory tasks, e.g., ratings of comprehensibility, memory, and speed of judging acceptability. The present study should extend the generality of these findings by relating the ordering of rule violations to the depth of 
Table 1

Examples of Meaningless Foils in Each of the Five Violation Conditions

Scrambled Sentence

Noon leave us to Harold by building told the. Student now south is former the in teaching his. Agent facts needed the secret urgently the possessed.

Multiple Violation

The swamp was not very virtuous for either candy. The dove will tell tires from sympathy to speak. Crooks and coffins refined it was a dirty zebra.

\section{Phrase Marker Violation}

Sam does not eraser to speak in public. Mr. Hobbs is a remember in our community. Anita asked us to use the collapse typewriter.

\section{Subcategorization Rule Violation}

Joe had not revealed with the professor's lecture. The dead and rotting tree floated the stagnant pond. The young girl abandoned in a trash can.

\section{Selectional Restriction Violation}

Nancy told the hurricane to bring a salad. Mr. Hill loaded the misery onto the waiting truck. My aunt never reads the daily bedspread.

comprehension required to detect them.

Depth of comprehension can be assessed by presence or absence of an "ambiguity effect." That is, conditions in which ambiguous sentences require longer than unambiguous sentences to be judged meaningful can be regarded as deeper than tasks in which no such difference in processing time for ambiguous and unambiguous sentences is found. The extra time is assumed to be taken up by ambiguity resolution; and comprehension is considered to be deeper when ambiguity is resolved than when it is not.

Three types of ambiguous stimulus sentence appear in the present study: lexical ambiguities, surface structural ambiguities, and underlying structural ambiguities. Lexical ambiguity occurs when a word or sequence of words has two distinct meanings and no differences at other grammatical levels. For instance, Marsha bought some beautiful new glasses is lexically ambiguous, since "glasses" can either be stemware or spectacles. The manner in which words can be grouped into phrases is represented in the surface structure of the sentences; ambiguity at the surface structure level involves the possibility of more than one grouping of adjacent words. For example, Interested faculty and students should not miss this talk, is ambiguous at the surface structure level. If the word "interested" is grouped with the phrase "faculty and students," then only faculty and interested students should attend; however, if the "interested" is grouped only with "faculty," then interested faculty and all students should attend. Finally, the underlying structural level of sentences represents the essential "logical" relations between words and phrases. In the two sentences: The president told his aides to prevent lying, and The president told his aides to quit lying, the logical relationship between "aides" and "lying" is quite different. Ambiguity at the underlying structural level involves neither a change in the meaning of the individual words nor a change in the grouping of words, but a change in the logical relations between the words. The sentence, The president told his aides to stop lying, is therefore ambiguous at the underlying structural level, since it leaves unclear who is doing the lying-the aides or somebody else.

These three types of ambiguity are related to processes which must be involved in sentence comprehension, and it is assumed that the ambiguities are resolved at the time the related process is performed. Lexical ambiguity is presumably resolved when meanings are assigned to the words in a sentence. Resolution of surface-structural ambiguity must occur when syntax is computed, that is, when the hearer determines the surface structure of the sentence. Underlying structural ambiguity is likely to be resolved when the logical relations in the sentence are extracted or when the "underlying structure" is ascertained. The present study may show the order in which lexical, surface structural, and underlying structural determinations are made in sentence comprehension, by manifesting the pattern of ambiguity resolution at progressively greater comprehension depths. If, for example, gross violations result in no ambiguity resolution, less gross violations result in only underlying structural ambiguity resolution, and the least gross violations result in resolution of both surface and underlying structural ambiguities, we will infer that underlying structural ambiguities are resolved at an earlier point, or in shallower comprehension, than underlying structural ambiguities. This would, in tum, suggest that underlying structure is determined before surface structure during sentence comprehension.

\section{METHOD}

\section{Design}

The design was a 5 by 4 by 2 by 2 factorial having one between-subject variable and three within-subject variables. Between subject was violation condition (scrambled, multiple violation, phrase marker violation, subcategorization rule violation, and selectional restriction rule violation). The three within-subject variables were ambiguity (unambiguous, lexical, surface structural, underlying structural); grammatical subject (common noun, proper noun); and transformation (affirmative, negative). The latter two variables were included for generality and counterbalancing.

\section{Subjects}

A total of 45 native English speakers, all paid student volunteers, served as subjects. An additional eight subjects were dropped from the study, four because of an excessive error rate ( $>15 \%)$ and four because of experimenter error or equipment malfunction. There were nine subjects per violation condition.

\section{Materials}

All subjects judged the same set of 144 meaningful sentences, which were interspersed with 72 meaningless foils appropriate to each violation condition. The meaingful sentences included 36 
each which were unambiguous, or ambiguous at the lexical, surface structural, or underlying structural level. These were ordered according to a blocking strategy to distribute practice and/or fatigue effects. All sentences were 7,8 , or 9 words long, with a mean sentence length of 8.2 words. Meaningful and meaningless sentences were randomly interspersed subject to the limitation that no more than four of either type of sentence could occur in a row. The overall strategy used in constructing distractor sentences involved altering an acceptable sentence in one of five ways. In the scrambled sentence condition, the words of a normal sentence were put in random order. In the multiple violation condition, an acceptable sentence was altered, largely by means of changing the content words with the view to making the sentence "weird." In the phrase-marker violation condition, one of the words of a normal sentence was changed to a word of a different part of speech, such that the altered sentence was unacceptable. Sentences in the subcategorization rule violation and the selectional restriction violation conditions were constructed by violating the subcategorization rules and selectional restrictions of English. Examples of distractor sentences in each condition can be found in Table 1. Distractor sentences were $7,8,9$, or 10 words long with a mean length of 8.0 words.

All sentences were typed on 5 by 8 cards and photographed. The resulting negatives, in which the lettering appeared white on a dark background, were mounted in 2 by 2 slide mounts for projection by slide projector.

\section{Apparatus and procedure}

The apparatus consisted of a slide projector, a voice-operated relay, two standard timers, and an interval timer. Subjects were tested individually. Sentences were projected on a rear viewing screen in a dimly-lighted room. The subject started each trial by pressing an advance key. This keypress opened the shutter in front of the projector, exposing the sentence and starting the timer. The subject judged the acceptability of the sentence by means of a vocal "yes" or "no" response. At the sound of the subject's voice, the shutter closed, the slide projector advanced, and the timer stopped. Latencies were recorded by hand, after which the experimenter activated a signal indicating that the subject was free to begin the next trial. Minimum intertrial interval was $3 \mathrm{sec}$, regulated by an automatic timer. Blank slides occurred every 15 trials, and the subject was given a brief rest period after 80 and 160 trials. Six practice trials were given to demonstrate the task, including three meaningful (unambiguous) sentences and three strings exemplifying the type of "meaningless" item to which subjects would be exposed throughout the experiment.

\section{RESULTS AND DISCUSSION}

Error rates differed among the five conditions, $F(4,40)=3.54, p<.025$. Errors tended to increase with longer latencies (see Table 2); thus the different error rates do not represent a speed-accuracy tradeoff.

Each meaningful sentence could be identified by the combination of factors comprising it: type of ambiguity, type of grammatical subject, and grammatical form (affirmative or negative). Each unique combination of factors occurred nine times for each subject. Because the hypothesis under test is that a strategy builds up as the subject discovers the nature of the stimulus materials, the first four occurrences of each combination of factors were considered as "warm-up" trials and the last five occurrences were included in the data analysis. Errors were dropped from the data, and the median of the
Table 2

Latency (in Seconds) to Respond in Each of the Various Experimental Conditions

\begin{tabular}{|c|c|c|c|c|c|c|}
\hline Meaning & 5 & Ambiouity & $\begin{array}{r}\text { Ser } \\
\text { Comn } \\
\text { Nou }\end{array}$ & $\begin{array}{l}\text { ntence } \\
\text { mon } \\
\text { un }\end{array}$ & $\begin{array}{r}\text { Subje } \\
\text { Pro } \\
\text { No } \\
\end{array}$ & \\
\hline Items & $(\%)$ & Type & Affirm & $\mathrm{Neg}$ & Affirm & Neg \\
\hline Scramble & ed Sent & nce & & & & \\
\hline & & Unambiguous & 2.4 & 2.5 & 2.5 & 2.3 \\
\hline & & Lexical & 2.6 & 2.3 & 2.3 & 2.7 \\
\hline 2.6 & 2.5 & Surface & 2.4 & 2.6 & 2.5 & 2.7 \\
\hline & & Underlying & 2.6 & 2.6 & 2.7 & 2.5 \\
\hline Multiple & Violat & & & & & \\
\hline & & Unambiguous & 2.7 & 2.2 & 2.5 & 2.4 \\
\hline & & Lexical & 2.5 & 2.1 & 2.3 & 2.5 \\
\hline 2.7 & 7.2 & Surface & 2.3 & 2.4 & 2.4 & 2.5 \\
\hline & & Underlying & 2.6 & 2.3 & 2.4 & 2.6 \\
\hline Phrase M & Iarker & iolation & & & & \\
\hline & & Unambiguous & 2.3 & 2.4 & 2.6 & 2.5 \\
\hline & & Lexical & 2.6 & 2.4 & 2.5 & 2.6 \\
\hline 2.6 & 3.7 & Surface & 2.5 & 2.7 & 2.6 & 2.8 \\
\hline & & Underlying & 3.0 & 2.8 & 2.7 & 2.7 \\
\hline Subcateg & gorizati & on Rule Violatio & & & & \\
\hline & & Unambiguous & 2.9 & 3.0 & 3.3 & 3.1 \\
\hline 35 & 80 & Lexical & 3.4 & 2.9 & 3.3 & 3.4 \\
\hline 3.5 & 8.0 & Surface & 3.2 & 3.3 & 3.2 & 3.4 \\
\hline & & Underlying & 3.4 & 3.6 & 3.5 & 3.6 \\
\hline Selection & nal Res & riction Violatior & & & & \\
\hline & & Unambiguous & 2.7 & 2.8 & 3.2 & 3.0 \\
\hline 3.1 & 5.3 & Lexical & 3.2 & 3.0 & 3.1 & 3.3 \\
\hline 3.1 & 5.3 & Surface & 3.0 & 3.6 & 3.3 & 3.4 \\
\hline & & Underlying & 3.6 & 3.5 & 3.6 & 3.3 \\
\hline
\end{tabular}

remaining scores for each combination of factors was computed and used as input to the analysis of variance. Only latencies to judge meaningful sentences were included in the analysis; however, mean latencies to judge meaningless foils in the five conditions appear in Table 2. In accordance with a suggestion by Clark (1973), two analyses of variance were computed. In the first analysis, subjects were considered as random variables and stimuli were considered as fixed effects. In this analysis, there was a main effect for violation, $F(4,40)=2.74, \quad p<.05$, and for ambiguity, $\mathrm{F}(3,120)=19.55, \mathrm{p}<.001$. The Ambiguity by Violation interaction was significant, $F(3,120)=2.24$, $p<.025$. The results are shown in Table 3 , which contains the means and SDs for the ambiguity effects in each of the five violation conditions and are further discussed below. Sentence subject was significant, $F(1,40)=9.25, p<.005$, reflecting the relative slowness of proper noun as compared to common noun subjects. A significant Ambiguity by Transformation interaction, $F(3,120)=5.64, \quad p<.005$, reflects the fact that affirmative sentences were most sensitive to surface structural ambiguities, while negative sentences were more sensitive to underlying structural ambiguities. Ambiguity by Sentence Subject by Transformation was significant, $F(3,120)=11.32, p<.001$. This interaction is not readily interpretable; however, it stems primarily 
Table 3

Means and Standard Deviations (in Seconds) for Ambiguity Effects in Each of the Violation Conditions

\begin{tabular}{|c|c|c|c|c|c|c|c|}
\hline \multicolumn{8}{|c|}{ Type of Ambiguity } \\
\hline \multicolumn{2}{|c|}{$\begin{array}{l}\text { Unambig- } \\
\text { uous }\end{array}$} & \multicolumn{2}{|c|}{ Lexical } & \multicolumn{2}{|c|}{$\begin{array}{l}\text { Surface } \\
\text { Structure }\end{array}$} & \multicolumn{2}{|c|}{$\begin{array}{l}\text { Underlying } \\
\text { Structure }\end{array}$} \\
\hline Mean & SD & Mean & SD & Mean & SD & Mean & SD \\
\hline \multicolumn{8}{|c|}{ Scrambled Sentence } \\
\hline 2.4 & .81 & 2.5 & .65 & 2.5 & .74 & 2.6 & .77 \\
\hline Multiple & Violat & & & & & & \\
\hline 2.4 & .53 & 2.3 & .59 & 2.4 & .67 & 2.5 & .68 \\
\hline \multicolumn{8}{|c|}{ Phrase Marker Violation } \\
\hline 2.5 & .45 & 2.5 & .43 & 2.6 & .50 & 2.8 & .61 \\
\hline \multicolumn{8}{|c|}{ Subcategorization Rule Violation } \\
\hline 3.1 & 1.16 & 3.2 & 1.11 & 3.3 & 1.02 & 3.5 & 1.31 \\
\hline \multicolumn{8}{|c|}{ Selectional Restriction Violation } \\
\hline 2.9 & .46 & 3.2 & .59 & 3.3 & .66 & 3.5 & .75 \\
\hline
\end{tabular}

from negative sentences containing lexical ambiguities, where proper nouns were much slower $(2.9 \mathrm{sec})$ than common nouns $(2.5 \mathrm{sec})$. In affirmative sentences, proper nouns were slightly faster $(2.7 \mathrm{vs} .2 .8 \mathrm{sec})$.

A second analysis of variance was computed treating stimuli as a random variable and subjects as a fixed effect. In this analysis, there was a main effect for violation, $F(4,256)=151.33, \mathrm{p}<.001$, for ambiguity, $\mathrm{F}(3,64)=3.45, \quad \mathrm{p}<.025$, and for Ambiguity by Sentence Subject by Transformation, $F(3,64)=3.08$, $\mathrm{p}<.05$. The Violation by Ambiguity interaction was not significant, $F(12,256)=1.29$. For those main effects which were significant in both analyses, min $F^{\prime}$ was computed. For Violation, min F' $(4,42)=2.69, p<.05$; for ambiguity min $F^{\prime}(3,85)=2.96, p<.05$. Min $F^{\prime}$ is an extremely conservative measure; significance levels for the true F's are probably nearer .01 .

Violation condition clearly influences processing speed, as evidenced by the violation condition main effect. Since all subjects in the study received the same set of meaningful sentences and only meaningful sentences were included in the statistical analyses, the differences in processing speed resulted from the nature of the meaningless sentences. This suggests that characteristics of the unanalyzed materials determined the latencies in the various experimental conditions. If different latencies reflect different amounts or types of processing, it appears that the subject's processing is partially determined by the distractor sentences. An important methodological question is thus raised: How can we know that the processing level adopted by the laboratory subject is representative of his or her extralaboratory information-processing of laboratory materials? Ultimately, there is no way to know for certain. However, the problem is apt to be most acute in experiments where the stimuli are so constructed that the subject may be able to infer the full set, i.e., when the entire set of stimuli is based on a small number of prototypes. In such studies, subjects may be able to infer the universe of materials to which they will be exposed and to adopt a processing strategy appropriate to those materials-which may be deeper or shallower than the processing mode they employ in similar tasks outside the laboratory with less predictable inputs. Materials in semantic memory studies are commonly constructed from a small number of prototypes, e.g., Collins and Quillian (1969), Meyer (1970), Meyer and Schvaneveldt (1971), Rips, Shoben and Smith (1973), Sherman (1973). Interestingly, Rips (Note 2) has found that a relatively minor variation in some of the stimuli in an experiment like Meyer's substantially alters the pattern of reaction times to other, unchanged stimuli.

Different latencies in the different violation conditions suggest different amounts or types of internal processing. It would be valuable to know the nature of the changed processing as the meaningfulness judgment ask became more demanding. Some suggestions may be derived from the nature of the Violation by Ambiguity interaction, however, the interpretation of this interaction must be done cautiously, since it has only been shown to generalize to a new population of subjects. While the effects of both violation condition and ambiguity have been shown reliable over both new subjects and new stimuli, it is conceivable that some property of this particular set of sentences determined the nature of their interaction, and the statistical analysis has not definitely ruled out this possibility. Nevertheless, several aspects of the interaction can be taken as suggestive of a number of important inferences regarding the internal information processing required in this study, even though we must use caution in generalizing to new stimuli.

The Violation by Ambiguity interaction reflects the hypothesized ambiguity effect in each of the between-subject violation conditions. Newman-Keuls analyses (based on variance analysis in which subjects was the only random variable) indicated that there was no ambiguity effect for either the scrambled sentence condition or the multiple violation condition. This is a partial replication of Mistler-Lachman (1972), since the meaningless foils in that study. were multiple violations. Evidently, the scrambled sentences and strings containing multiple linguistic violations in this study could be discriminated from English sentences without resolution of any ambiguities contained in the English sentences. In other words, it was possible to distinguish meaningful from meaningless sentences without assigning an unambiguous reading to any of them. Thus, indeed, these two tasks were apparently effected with shallow processing. Newman-Keuls analyses indicate that in the phrase marker violation condition, only one form of ambiguity was significantly slower than unambiguous sentences-underlying structural ambiguities. The same was found true in the subcategorization rule violation condition. In the selectional restriction violation condition, Newman-Keuls analyses indicated that all 
three types of ambiguity were significantly slower than unambiguous sentences. It appears that the violation conditions in this study did introduce progressively deeper comprehension. There was no ambiguity effect in the scrambled sentence and multiple violation condition; subjects in these conditions were performing the task at a shallow level of comprehension. Only underlying structural ambiguities had an effect in the phrase marker violation and subcategorization rule violation conditions; thus, subjects in these conditions were performing the task at an intermediate level of comprehension. Finally, all three types of ambiguity were effective in the selectional restriction violation condition; subjects in this condition were required to understand the sentence deeply in order to distinguish sentences containing such violations from acceptable sentences. Several conclusions of interest follow from these findings.

First, the depth of comprehension as inferred from the ambiguity effect was not directly related to latencies. It is possible for two experimental conditions to result in similar overall latencies, but to result in very different inferences about comprehension depth from the ambiguity effect. The phrase marker violation condition and the subcategorization rule violation condition apparently required equal comprehension depth, in that both required resolution only of underlying structural ambiguity. No ambiguity effect was found in the multiple violation condition, suggesting shallower comprehension. However, latencies in the phrase marker violation condition were much closer to those in the multiple violation condition than they were to latencies in the subcategorization rule violation condition. This was true both for time to respond "yes" and time to respond "no." This outcome is more consistent with Craik and Lockhart's (1972) position than with that of multistore models. Craik and Lockhart view depth of processing as an encoding level which characterizes processing from the onset; whereas, it appears that multistore models would view additional depth as additional steps appended to processing after the early stages are complete. To the extent that such appended processes are serial or partly serial they should add to processing time. Thus, a multistore model should predict that deeper processing takes longer. Since similar latencies were found in tasks requiring different depths of comprehension, the present results are more consistent with the Craik and Lockhart view.

A second finding of interest involved the emergence of different types of ambiguity resolution. Violations of less severity resulted in slower processing of ambiguities, as expected. This effect was cumulative; first underlying structural ambiguities slowed processing, then surface and lexical ambiguities. No reversals occurred, as would have happened for example if no effect of underlying structural ambiguities had been found in the selectional restriction violation condition. This permits an ordering of the lasks in this study into three levels of comprehension: shallow (scrambled sentence, multiple violation), intermediate (phrase marker violation, subscategorization rule violation), and deep (selectional restriction violation). If it is inferred that the slowing of processing resulted from resolution of the ambiguity, then at intermediate levels of comprehension underlying structural ambiguities were resolved while surface structural and lexical ambiguities were not. If we may relate the resolution of underlying structural ambiguities to discovery of the logical relationships involved in a sentence, then we may conclude that discovery of these relationships "precedes" discovery of the precise syntactic structure of the sentence and the determination of word meanings. The meaning of "precedes" differs in multistore and levels-of-processing analyses; for the multistore theorist, "precedes" might imply "occurs earlier." For the levels-of-processing theorist, it need only imply "occurs in shallower encoding." Put another way, there are some encoding levels which require computation of logical relationships but not computation of syntax; but if computation of syntax is required, then logical relationships must be computed also.

While it might at first seem counterintuitive to suggest that computation of logical relationships precedes, rather than depends upon, computation of surface grammatical relationships, in fact it accords with common linguistic experience. It is a commonplace that one can understand more of a partially learned foreign language than he can produce; apparently logical relationships can be extracted before the surface structural rules are fully learned. Pidgin languages rely for their success precisely on excluding the niceties of surface structure-they utilize enough rules to represent the logical relationships among content words and little else. The precedence of underlying structure also accords with theoretical considerations. Anderson and Bower (1973) report considerable difficulty in constructing a computer program to parse the surface structure of a linguistic string without foreknowledge of the underlying structure. MacNamara (1972) argues that children learn surface structures by relating them to known meanings. Thus, it may be that comprehension involves hypothesizing an underlying structure without full parsing, performing a full syntactic analysis only when it is necessary. Since there are fewer underlying structures in a language than surface structures, and since, in normal discourse, these are more predictable than surface forms, this would be a highly efficient comprehension process.

Finally, it may seem odd that underlying structural relationships are computed prior to lexical meanings. This outcome may reflect particular problems in processing lexical ambiguity noted by others (Foss, Bever, \& Silver, 1968). Or perhaps a certain amount of meaning is computed very early to aid the computation of logical relationships, but ambiguity resolution 
requires a return of meaning determination at the lexical level.

\section{REFERENCES}

Anderson, J. R.. \& Bower, G. H. Human associative memory. Washington, D.C: V. H. Winston \& Sons, 1973.

Chomsky, N. Aspects of the theory of syntax. Cambridge: MIT Press, 1965.

Clark, H. H. The language-as-fixedeffect fallacy: A critique of language statistics in psychological research. Joumal of Verbal Learning and Verbal Behavior, 1973, 12, 335-359.

Collins, A. M., \& Quillian, M. R. Retrieval time from semantic memory. Joumal of Verbal Leaming and Verbal Behavior, $1969,8,240-247$.

Craik, F. I. M. \& Lockhart, R. S. Levels of processing: A framework for memory research. Journal of Verbal Learning and Verbal Behavior, 1972, 11, 671-684.

Foss, D., Bever, T., \& Silver, $M$. The comprehension and verification of ambiguous sentences. Perception \& Psychophysics, 1968, 4, 304-306.

MacNamara, J. Cognitive basis of language learning in infants. Psychological Review, 1972, 79, 1-13.

Meyer, D. E. On the representation and retrieval of stored semantic information. Cognitive Psychology, 1970, 1 , 242-300.

Meyer, D. E., \& Schvaneveldt, R. W. Facilitation in recognizing pairs of words: Evidence of a dependence between retrieval operations. Joumal of Experimental Psychology, 1971, 90 , $227-234$.

Mistler-Lachman, J. Levels of comprehension in processing of normal and ambiguous sentences. Journal of Verbal Learning and Verbal Behavior, 1972, 11, 614-623.

Moore, T. E. Speeded recognition of ungrammaticality. Journal of Verbal Learning and Verbal Behavior, $1972,11,550-560$.

Rips, L. J., Shoben, E. J., \& Smith, E. E. Semantic distance and the verification of semantic relations. Journal of Verbal Learning and Verbal Behavior, 1973, 12, 1-20.

Sherman, M. A. Bound to be easier? The negative prefix and sentence comprehension. Journal of Verbal Learning and Verbal Behavior, $1973,12,76-84$

Stolz, W. Some experiments with queer sentences, Language and Speech, $1969,12,203-219$.

\section{REFERENCE NOTES}

1. Hamilton, H. W., \& Deese, J. Imagery and grammaticality in the service of comprehensibility. Unpublished manuscript, University of Maryland, 1973.

2. Rips, L. J. Quantification and semantic memory. Unpublished manuscript, Stanford University, 1973.

(Received for publication January 28, 1974; revision received September $30,1974$. ) 